\title{
Optimizing insulin initiation in primary care: the Diabetes CoStars patient support program
}

This article was published in the following Dove Press journal:

Pragmatic and Observational Research

28 January 2016

Number of times this article has been viewed

\section{Olga K Lutzko' \\ Helen Schifferle ${ }^{2}$ \\ Marita Ariola ${ }^{3}$ \\ Antonia Rich ${ }^{4}$ \\ Khen Meng Kon ${ }^{5}$}

'Kardinia Health, GP Super Clinic, Belmont, VIC, ${ }^{2}$ Medicross Rothwell, Rothwell, QLD, ${ }^{3}$ Innerwest Specialist Centre, Burwood, NSW, Australia; ${ }^{4}$ Atlantis Healthcare Pty Ltd, Sydney, Australia; ${ }^{5}$ Sanofi Australia Pty Ltd, Macquarie Park, NSW, Australia
Correspondence: Olga K Lutzko Kardinia Health, GP Super Clinic, 2-18 Colac Rd, Belmont, VIC 3216, Australia

Tel +6I 431946898

Email olutzko@gmail.com
Purpose: The purpose of this study was to evaluate the optimization of fasting blood glucose (FBG) levels in patients with type 2 diabetes mellitus newly initiated on insulin glargine who were enrolled in the Australian Diabetes CoStars Patient Support Program (PSP).

Patients and methods: A retrospective analysis of data from 514 patients with type 2 diabetes mellitus who completed the 12-week Diabetes CoStars PSP was performed. All patients were initiated on insulin glargine in primary care and enrolled by their general practitioner, who selected a predefined titration plan and support from a local Credentialled Diabetes Educator. The data collected included initial and final insulin dose, self-reported FBG, and glycated hemoglobin $\left(\mathrm{A}_{1 \mathrm{c}}\right)$ levels.

Results: The insulin dose increased in $81 \%$ of patients. Mean FBG was reduced from $208.8 \mathrm{mg} / \mathrm{dL}(11.6 \mathrm{mmol} / \mathrm{L})$ to $136.8 \mathrm{mg} / \mathrm{dL}(7.6 \mathrm{mmol} / \mathrm{L})$ after 12 weeks. Initial and final $A_{1 c}$ values were available for 99 patients; mean $A_{1 c}$ was reduced from $9.5 \%(80 \mathrm{mmol} / \mathrm{mol})$ to $8.1 \%(65 \mathrm{mmol} / \mathrm{mol})$. The reductions in mean $\mathrm{FBG}$ and $\mathrm{A}_{1 \mathrm{c}}$ were similar irrespective of titration plan. Overall, $27.2 \%$ of patients achieved FBG levels within the titration plan target range of $72-108 \mathrm{mg} / \mathrm{dL}$ (4-6 mmol/L) and an additional $43.4 \%$ of patients achieved FBG within the range recommended by current Australian guidelines $(110-144 \mathrm{mg} / \mathrm{dL}[6.1-8.0 \mathrm{mmol} / \mathrm{L}])$. Overall, $23.3 \%$ of patients achieved the $\mathrm{A}_{1 \mathrm{c}}$ target of $\leq 7 \%$.

Conclusion: These data demonstrate that the majority of patients enrolled in the Diabetes CoStars PSP achieved acceptable FBG levels 12 weeks after starting insulin therapy irrespective of titration plan.

Keywords: insulin glargine, titration, type 2 diabetes mellitus, fasting blood glucose

\section{Introduction}

In the face of a rising global prevalence of diabetes, estimated at $9 \%$ in 2014, ${ }^{1}$ primary care physicians are becoming increasingly responsible for the initiation of insulin therapy in patients with type 2 diabetes mellitus (T2DM). However, failure to initiate and intensify insulin therapy when indicated (clinical inertia) is a problem around the world. ${ }^{2-5}$ Australian patients with T2DM are initiated on insulin therapy after an average diabetes duration of 8.1 years and with glycated hemoglobin $\left(\mathrm{A}_{1 \mathrm{c}}\right)$ levels of $8.2 \%$ (66 $\mathrm{mmol} / \mathrm{mol})$ to $9.4 \%$ (79 $\mathrm{mmol} / \mathrm{mol})$, significantly above the widely recommended target $\mathrm{A}_{1 \mathrm{c}}$ level of $\leq 7 \%$ ( $\left.\leq 53 \mathrm{mmol} / \mathrm{mol}\right)$, suggesting that many patients spend too long with suboptimal blood glucose control. ${ }^{6,7}$

An important barrier to insulin therapy in primary care is the perceived complexity of insulin initiation and titration by primary care physicians. ${ }^{8,9}$ Moreover, because insulin therapy has traditionally been initiated by specialists, primary care physicians may lack the knowledge and experience required to take on the role of insulin initiation, as well as the time required to educate patients. ${ }^{8,10-12}$ 
Patients starting insulin therapy often require education and support beyond that which can typically be provided by primary care physicians. As specialists in their field, Credentialled Diabetes Educators (CDEs) are well positioned to provide patients with the self-management education and support that is an essential component of diabetes care. ${ }^{13,14}$ CDE-led patient support has been shown to improve patient outcomes in primary and secondary care settings. ${ }^{15-17}$

The Australian Diabetes CoStars Patient Support Program (PSP) is designed to assist in the optimization of blood glucose levels using an appropriate titration plan for adult patients with T2DM newly initiated on insulin glargine, a long-acting basal insulin analog, in primary care. The 12-week program provides CDE-led titration support, educational materials, and motivational assistance for patients. Patients are enrolled into the PSP by their general practitioner (GP), who selects a predefined titration plan and the level of patient support required. The PSP recognizes the importance of simplifying insulin initiation for the primary care physician by providing a clear protocol for titration and a model of care where patient education and titration support is led by a CDE..$^{8,18,19}$ The Diabetes CoStars PSP was started in Australia in August 2012; this retrospective analysis of data from patients with T2DM enrolled in Diabetes CoStars was performed to evaluate glycemic control after insulin initiation in the primary care setting among program participants.

\section{Methods}

\section{Study design}

This study was a retrospective analysis of preexisting data collected by the Diabetes CoStars PSP between October 9, 2012 and February 13, 2014. This study was reviewed and approved by the Bellberry Human Research Ethics Committee.

\section{Patients}

All Australian adult patients being newly initiated on insulin glargine are eligible to enroll in the Diabetes CoStars PSP. All eligible and enrolled patients who had provided written informed consent for the use of their data and completed the 12-week Diabetes CoStars PSP were included in the analysis. Enrolled patients were assigned to one of three adapted titration plans (Plan A, B, or C; Figure 1) $)^{19-22}$ by their GP and also provided with materials and resources covering different aspects of living with diabetes. The GP selected the level of support required - general support (via TeleHealth) and/or free sessions with a local CDE. Sessions with the local CDE comprised of either one face-to-face consultation and four phone calls (stream 1) or two face-to-face consultations and two phone calls (stream 2), as decided by the CDE in conjunction with the patient. Regardless of the titration plan selected, patients were supported to titrate to the right dosage of daily insulin glargine required to manage their diabetes. Patients who received TeleHealth only support and patients who received one-off training on how to use their insulin glargine pen delivery system were excluded from the analysis.

\section{Data collection}

The data were collected from an electronic database managed by Atlantis Healthcare in Australia. Sex, titration plan (A, B, or C), CDE stream allocation (one or two face-to-face sessions), and clinical parameters (initial and final insulin dose, self-reported fasting blood glucose $[\mathrm{FBG}], \mathrm{A}_{1 \mathrm{c}}$ levels

\section{Plan A: preset titration}

Plan B: preset titration

Adjust dose weekly to achieve FBG target
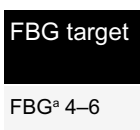

$\mathrm{mmol} / \mathrm{L}^{19,20}$ of $4-6 \mathrm{mmol} / \mathrm{L}^{19,21,22}$

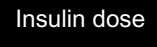

Increase daily

dose by $2 \mathrm{U}$

every 3 days $^{\text {b }}$ to achieve FBG target ${ }^{19,20}$

\begin{tabular}{|l|l|l|l|}
\hline $\begin{array}{l}\text { Mean FBG } \\
(\mathrm{mmol} / \mathrm{L})\end{array}$ & $\begin{array}{l}\text { Change in } \\
\text { insulin dose }\end{array}$ & FBG target & $\begin{array}{l}\text { Change in } \\
\text { insulin dose }\end{array}$ \\
\hline$<4$ & $\begin{array}{l}\text { Reduce by } 2 \text { to } \\
4 \mathrm{U}^{\mathrm{c}}\end{array}$ & $\begin{array}{l}\text { Individualized } \\
\text { FBG target to be } \\
\text { defined by } \\
\text { physician }\end{array}$ & $\begin{array}{l}\text { Customized } \\
\text { titration } \\
\text { regimen to be } \\
\text { prescribed by } \\
\text { physician } \\
\text { (specify plan on } \\
\text { application } \\
\text { form) }\end{array}$ \\
\hline $6-5.9$ & No change & $+2 \mathrm{U}$ & \\
\hline $7-7.9$ & $+4 \mathrm{U}$ & & \\
\hline $8-10$ & $+6 \mathrm{U}$ & & \\
\hline$>10$ & $+8 \mathrm{U}$ & & \\
\hline
\end{tabular}

\section{Plan C: custom titration}

Figure I Titration plan options in the Diabetes CoStars Patient Support Program.

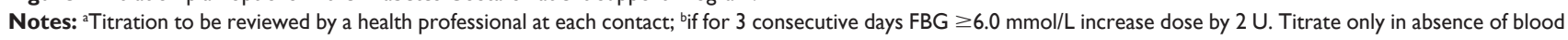
glucose level $(B G L)<4.0 \mathrm{mmol} / \mathrm{L}$. If $\mathrm{FBG}<4.0 \mathrm{mmol} / \mathrm{L}$ adjust insulin dose at clinician's discretion; cinsulin dose may be reduced (small increments of $2-4 \mathrm{U}$ ) in the presence of severe hypoglycemia (requiring assistance), or BLG $<3.0 \mathrm{mmol} / \mathrm{L}$ in the preceding week. Do not increase insulin dose if fasting $B G L<4.0 \mathrm{mmol} / \mathrm{L}$ any time in the preceding week. Information sourced from Davies et al, ${ }^{19}$ Diabetes Australia, ${ }^{20}$ Phillips, ${ }^{21}$ Riddle et al. ${ }^{22}$

Abbreviation: FBG, fasting blood glucose. 
[if available], and any hypoglycemic events reported to a $\mathrm{CDE})$ were collected.

\section{Statistical analysis}

No formal sample size calculation was undertaken and no formal hypothesis test was performed. The data extracted from the database were analyzed using SAS (V9.3). There were no imputations or replacements for missing data. Insulin dose, $\mathrm{FBG}$, and $\mathrm{A}_{1 \mathrm{c}}$ levels were analyzed using descriptive statistics and were presented overall as well as by titration plan and CDE stream allocation. The proportion of patients achieving target FBG levels was calculated according to the FBG target specified in the titration plans $(72-108 \mathrm{mg} / \mathrm{dL}$ [4-6 mmol/L]), which was based on Australian guidelines that were current during the time of data collection. ${ }^{20}$ These guidelines were updated after data collection and prior to manuscript preparation; the FBG target in the current guidelines is $108-144 \mathrm{mg} / \mathrm{dL}(6-8 \mathrm{mmol} / \mathrm{L}) .{ }^{13}$ The decision was made after data collection also to calculate the proportion of patients who achieved FBG levels within the current target range, which reflects the National Evidence-Based Guidelines for Blood Glucose Control in Type 2 Diabetes. ${ }^{23}$ Change in FBG was analyzed using a general linear model with change in FBG as the outcome variable and titration regime and $\mathrm{CDE}$ stream allocation as factors reflecting the plan followed as well as baseline FBG, initial insulin dose, and sex as patient relevant covariates. A logistic regression model was fitted to explore factors associated with the attainment of self-reported FBG within the target range (72-108 mg/dL [4-6 mmol/L]) for patients whose FBG was not within target at the commencement of the program using within target at the end of program (yes/no) as the outcome variable. Formal analyses were not conducted on spontaneously reported hypoglycemic events due to the low number of events reported.

\section{Results}

\section{Patient characteristics}

A total of 514 patients met the criteria for inclusion. Of these 161 were assigned to titration Plan A, 162 to titration Plan B, and 191 to titration Plan C. Mean self-reported FBG and mean insulin dose at initiation were similar overall and in each titration group (Table 1). Initial FBG ranged from 54 to $522 \mathrm{mg} / \mathrm{dL}$ (3-29 mmol/L) and initial insulin dose ranged from 2 to $120 \mathrm{U}$. An initial $A_{1 c}$ level was available for 234 patients; there was no significant difference in initial $\mathrm{A}_{1 \mathrm{c}}$ level overall and across the three titration groups (Table 2). $\mathrm{A}_{1 \mathrm{c}}$ values ranged between $5.7 \%(39 \mathrm{mmol} / \mathrm{mol})$ and $16.0 \%(151 \mathrm{mmol} / \mathrm{mol})$.
Table I Patient characteristics, overall, and by titration plan

\begin{tabular}{|c|c|c|c|c|}
\hline & Overall & $\begin{array}{l}\text { Plan A: } \\
\text { preset; } \\
2 \text { U/3 days }\end{array}$ & $\begin{array}{l}\text { Plan B: } \\
\text { preset; } \\
\text { weekly }\end{array}$ & $\begin{array}{l}\text { Plan C: } \\
\text { physician- } \\
\text { led }\end{array}$ \\
\hline n (\%) & $514(100)$ & I6I (3I.3) & $162(3 \mid .5)$ & I91 (37.2) \\
\hline Male (\%) & $297(57.8)$ & $92(57.1)$ & $96(59.3)$ & $109(57.1)$ \\
\hline $\begin{array}{l}\text { Mean FBG, } \\
\mathrm{mg} / \mathrm{dL}(\mathrm{SD})\end{array}$ & $209(65)$ & $211.3(73.1)$ & $209.7(60.7)$ & 205.7 (6I.7) \\
\hline $\mathrm{mmol} / \mathrm{L}(\mathrm{SD})$ & II.6 (3.6) & II.7 (4.1) & II.7 (3.4) & II.4 (3.4) \\
\hline $\begin{array}{l}\text { Mean insulin } \\
\text { dose, U (SD) }\end{array}$ & $20.7(20.0)$ & $18.4(16.5)$ & $23.4(22.8)$ & $20.4(20.0)$ \\
\hline
\end{tabular}

Abbreviation: FBG, fasting blood glucose.

\section{Patient outcomes}

The majority of patients in each titration group had their insulin dose increased over the 12-week program (Table 3). The dose increase was high in the group of patients following titration Plan A, followed by Plan B and Plan C.

Mean self-reported FBG level was similar overall and in each titration group by the end of the 12-week program (Figure 2). There was an overall mean reduction in FBG of $72 \pm 3.6 \mathrm{mg} / \mathrm{dL}(4.0 \pm 0.2 \mathrm{mmol} / \mathrm{L})$. The reduction in FBG was similar in each titration group: Plan A $(-77 \pm 6 \mathrm{mg} / \mathrm{dL}[-4.3 \pm 0.3$ $\mathrm{mmol} / \mathrm{L}], \mathrm{n}=154)$, Plan B $(-74 \pm 5 \mathrm{mg} / \mathrm{dL}[-4.1 \pm 0.3 \mathrm{mmol} / \mathrm{L}]$, $\mathrm{n}=159)$, and Plan C $(-66 \pm 4 \mathrm{mg} / \mathrm{dL}[-3.7 \pm 0.2 \mathrm{mmol} / \mathrm{L}]$, $\mathrm{n}=189$ ).

The proportion of patients who achieved FBG levels within the titration protocol target range of $72-108 \mathrm{mg} / \mathrm{dL}$ (4-6 mmol/L) after 12 weeks was similar across the three titration groups and overall (Figure 3). More patients achieved FBG levels within the range recommended by current guidelines updated after data collection $(110-144 \mathrm{mg} / \mathrm{dL}[6.1-8.0 \mathrm{mmol} / \mathrm{L}])^{13}$ than within the protocol target range, both overall and across the three titration groups (Figure 3). Overall, $70.6 \%$ of patients had FBG levels within one of the target ranges (between 72 and 144 $\mathrm{mg} / \mathrm{dL}$ [4-8 $\mathrm{mmol} / \mathrm{L}]$ ).

The overall mean $\mathrm{A}_{1 \mathrm{c}}$ level after 12 weeks was similar overall and in each titration group (Figure 4). Based on 99 patients for whom initial and final $A_{1 c}$ values were available, the overall mean reduction in $\mathrm{A}_{1 \mathrm{c}}$ was $1.3 \% \pm 0.2 \%$. A similar proportion

Table 2 Initial $A_{l c}$ level, overall and by titration plan

\begin{tabular}{lllll}
\hline & Overall & $\begin{array}{l}\text { Plan A: } \\
\text { preset; } \\
\text { 2 U/3 days }\end{array}$ & $\begin{array}{l}\text { Plan B: } \\
\text { preset; } \\
\text { weekly }\end{array}$ & $\begin{array}{l}\text { Plan C: } \\
\text { physician-led }\end{array}$ \\
\hline $\mathrm{n}(\%)$ & $234(100)$ & $83(35.5)$ & $78(33.3)$ & $73(31.2)$ \\
Mean $\mathrm{A}_{1 \mathrm{c}}$, & $9.5(1.7)$ & $9.4(1.8)$ & $9.4(1.5)$ & $9.6(1.9)$ \\
$\%(\mathrm{SD})$ & & & & \\
\hline
\end{tabular}

Abbreviations: $A_{1 c}$, glycated hemoglobin; SD, standard deviation. 
Table 3 Change in insulin dose after 12 weeks, overall (all patients), and by titration plan

\begin{tabular}{|c|c|c|c|c|}
\hline & Overall & $\begin{array}{l}\text { Plan A: } \\
\text { preset; } \\
2 \text { U/3 days }\end{array}$ & $\begin{array}{l}\text { Plan B: } \\
\text { preset; } \\
\text { weekly }\end{array}$ & $\begin{array}{l}\text { Plan C: } \\
\text { physician-led }\end{array}$ \\
\hline $\mathrm{n}$ & 508 & 158 & 160 & 190 \\
\hline \multicolumn{5}{|c|}{$\begin{array}{l}\text { Proportion of patients with increased, decreased, and } \\
\text { unchanged insulin dose, } n(\%)\end{array}$} \\
\hline Dose increase & $410(80.7)$ & $129(81.6)$ & $136(85.0)$ & $145(76.3)$ \\
\hline Dose reduction & $4 I(8.1)$ & $16(10.1)$ & $5(3.1)$ & $20(10.5)$ \\
\hline Dose unchanged & 57 (II.2) & $13(8.2)$ & $19(11.9)$ & $25(13.2)$ \\
\hline \multicolumn{5}{|c|}{ Change in insulin dose, $U$} \\
\hline Mean (SEM) & II.5 (0.7) & I4.I (I.4) & | 3.8 (I.4) & $7.4(0.9)$ \\
\hline
\end{tabular}

Abbreviation: SEM, standard error of the mean.

of patients achieved target $\mathrm{A}_{1 \mathrm{c}} \leq 7 \%(53 \mathrm{mmol} / \mathrm{mol})$ overall and in each titration group (Figure 3 ).

\section{Factors affecting achievement of target FBG}

Initial FBG level was the only factor identified as a predictor of achievement of the protocol FBG target. Although higher initial FBG values correlated with greater falls in FBG by the end of the 12-week program, the odds of achieving the protocol FBG target decreased by $13 \%$ for every $1 \mathrm{mmol} / \mathrm{L}$ increase in initial FBG (odds ratio $0.87,95 \%$ confidence interval: $0.81,0.93$ ).

\section{Spontaneously reported adverse events}

Adverse events were not specifically solicited during the study period. There were spontaneous reports of two episodes of hypoglycemia and four adverse events (other than hypoglycemia). A 73-year-old female patient died from an aggressive cancer 7 months after commencing insulin therapy; limited information was available and causality was not reported.

\section{Discussion}

This retrospective analysis of data from the Australian Diabetes CoStars PSP demonstrates that approximately one quarter of enrolled patients achieved their FBG target 12 weeks after initiation of insulin glargine. Enrolled patients followed a titration plan selected by their GP, two of which specified an FBG target range of 72-108 $\mathrm{mg} / \mathrm{dL}$ (4-6 mmol/L) based on Australian guidelines that were current at the time of data collection. Recommended blood glucose targets are based on levels that correlate with $\mathrm{A}_{1 \mathrm{c}}$ levels associated with a low risk of developing diabetes-related complications, given that $A_{1 c}$ level is the strongest predictor of diabetes-related complications. The titration plan FBG target is a stringent target that reflects normal blood glucose levels (normoglycemia) and correlates with $A_{1 c}$ levels associated with the lowest absolute risk of diabetes-related complications. ${ }^{24-26}$

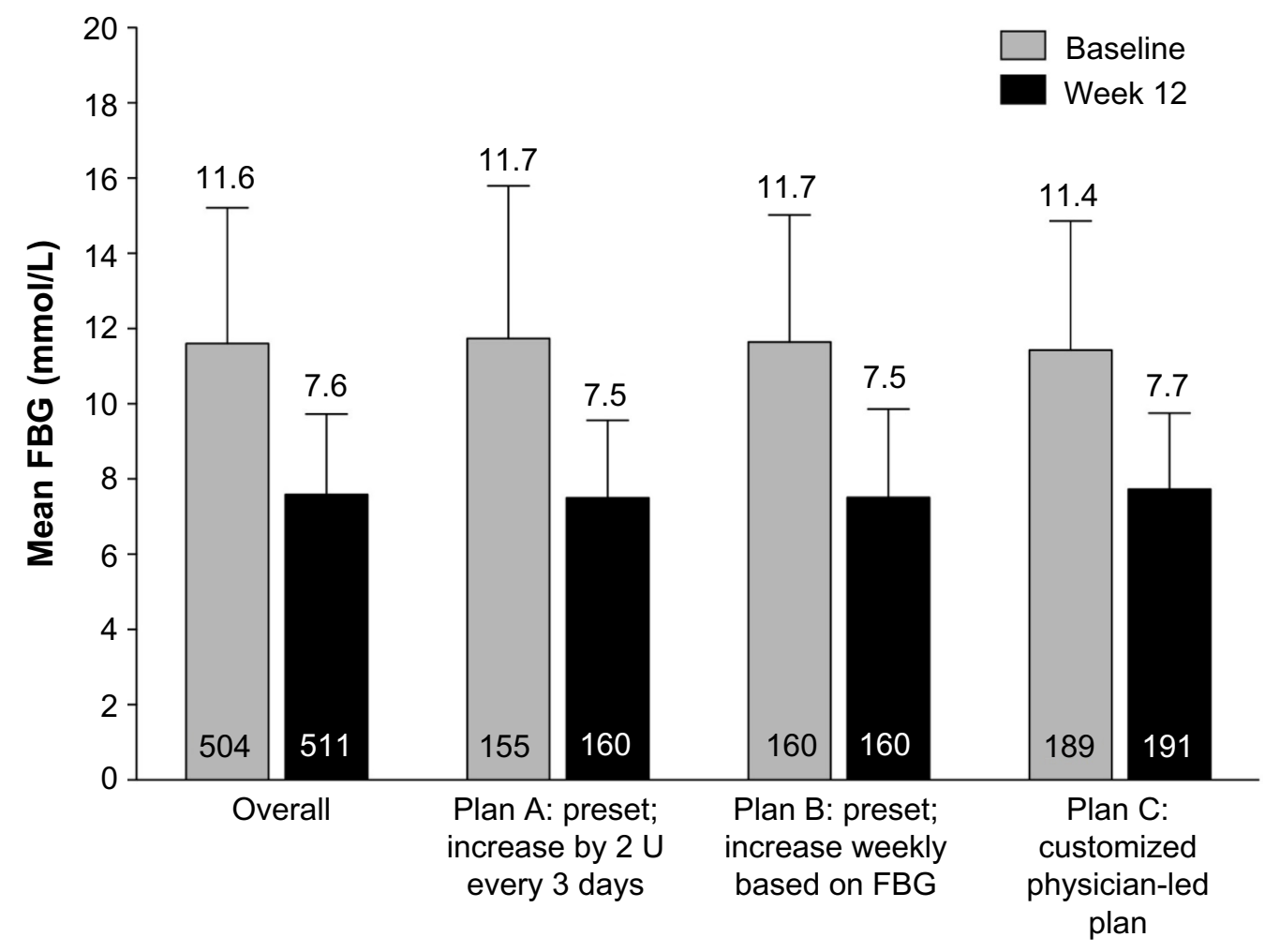

Figure 2 Mean change from baseline to week 12 in mean fasting blood glucose (FBG), overall (all patients), and by allocated titration plan. Notes: Error bars represent standard deviation. Numbers in bars are patient numbers. 


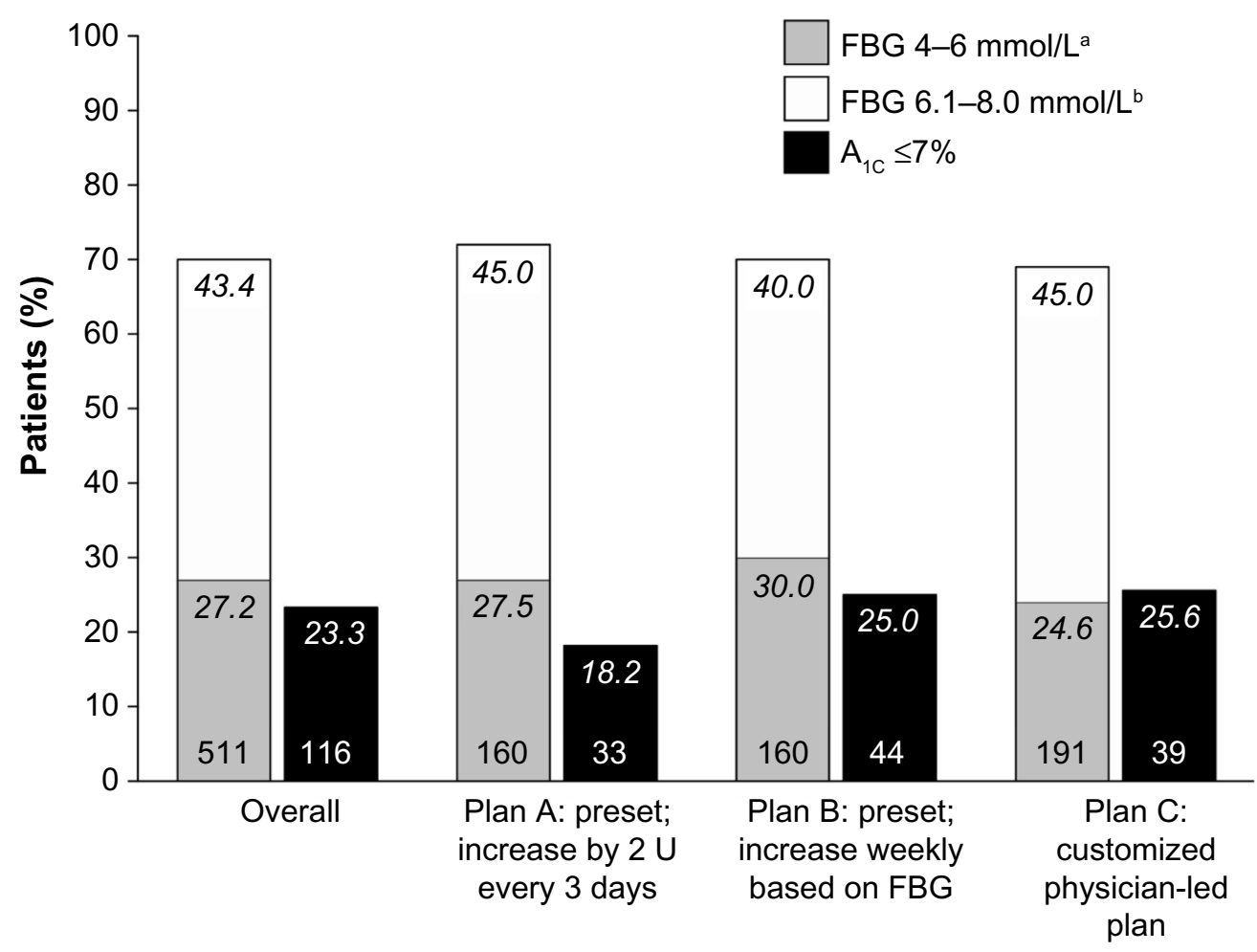

Figure 3 Proportion of patients with FBG and $A_{1 c}$ levels within target ranges 12 weeks after initiation of insulin therapy, overall (all patients), and by allocated titration plan. Notes: Numbers in bars are total patient numbers (non-italicized) and percent of patients (italicized). Patients were classified as achieving FBG $72-108 \mathrm{mg} / \mathrm{dL}$ (4-6 mmol/L) if their FBG level at the end of week 12 was $\geq 4$ and $\leq 6$, or FBG $110-144 \mathrm{mg} / \mathrm{dL}(6.1-8.0 \mathrm{mmol} / \mathrm{L})$ if their FBG level at the end of week 12 was $\geq 6$. I and $\leq 8.0$. ${ }^{\mathrm{a}} \mathrm{Titration}$

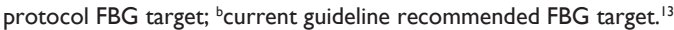

Abbreviations: $A_{1 c}$, glycated hemoglobin; FBG, fasting blood glucose.

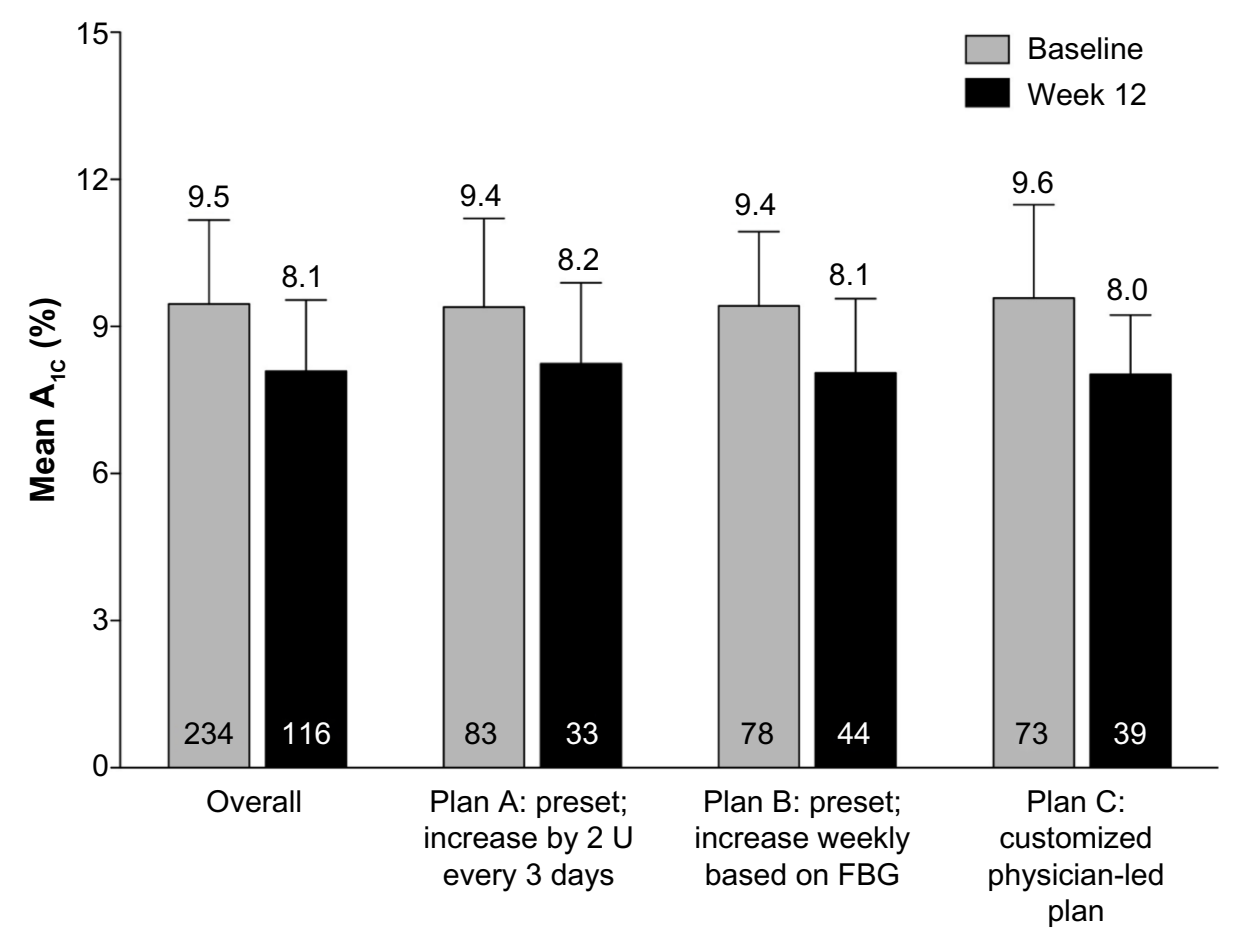

Figure 4 Mean change from baseline to week 12 in mean $A_{l c}$, overall (all patients), and by allocated titration regimen. Notes: Error bars represent standard deviation. Numbers in bars are patient numbers.

Abbreviations: $\mathrm{A}_{\mathrm{Ic}}$, glycated hemoglobin; FBG, fasting blood glucose. 
However, normoglycemia may not be a reasonable target for many patients, for example, when there is a high risk of hypoglycemia, limited life expectancy, advanced microvascular or macrovascular complications, extensive comorbid conditions, or long-standing diabetes where goals have been difficult to attain despite appropriate medical and multidisciplinary interventions. ${ }^{14}$ The Australian guidelines were updated in 2015 and currently recommend an FBG target range of $108-144 \mathrm{mg} / \mathrm{dL}(6-8 \mathrm{mmol} / \mathrm{L})$ to reflect these factors. ${ }^{13}$ When data were reported to reflect the current recommendations, it was found that approximately four in ten patients achieved FBG levels within a range of 110-144 $\mathrm{mg} / \mathrm{dL}(6.1-8.0 \mathrm{mmol} / \mathrm{L})$. Collectively, seven in ten patients enrolled in the Diabetes CoStars PSP achieved acceptable FBG levels between 72 and $144 \mathrm{mg} / \mathrm{dL}$ (4-8 mmol/L).

Patients with higher initial FBG levels at the time of insulin initiation were less likely to achieve FBG within the target range of 108-144 mg/dL (4-6 mmol/L), even though higher initial FBG level correlated with greater change in FBG over 12 weeks. Similarly, higher baseline levels of $A_{1 c}$ have been found to correlate with greater reductions in $A_{1 c}$ following CDE-led educational interventions, ${ }^{15}$ but also to reduce the likelihood of achieving target $A_{1 c}$ levels with insulin therapy ${ }^{27,28}$ These findings reinforce the importance of avoiding delayed treatment of hyperglycemia, including initiating insulin when oral antidiabetic agents and lifestyle measures no longer adequately control blood glucose levels. ${ }^{29,30}$

While approximately only one quarter of patients achieved the recommended $\mathrm{A}_{1 \mathrm{c}}$ target of $\leq 7 \%$ ( $\left.\leq 53 \mathrm{mmol} / \mathrm{mol}\right)$, each $1 \%$ reduction in $\mathrm{A}_{1 \mathrm{c}}$ has been shown to reduce the risk for microvascular complications by $37 \%$, for any end point related to diabetes by $21 \%$, for death related to diabetes by $21 \%$, and for myocardial infarction by $14 \% .{ }^{24}$ Although the rate of patients achieving target $\mathrm{A}_{1 \mathrm{c}}$ needs to be improved, the mean reduction in $\mathrm{A}_{1 \mathrm{c}}$ by $1.28 \%$ likely represents improved outcomes for many patients.

There are limited real world data available on glycemic control in patients with T2DM starting insulin therapy in the Australian primary care setting. The Australian Fremantle Diabetes Study is a large community-based observational study of a cohort of patients with T2DM followed up annually between 1993 and 2001. Median FBG and $A_{1 c}$ in the Fremantle cohort were reduced from $219.6(12.2 \mathrm{mmol} / \mathrm{L})$ to $163.8 \mathrm{mg} / \mathrm{dL}(9.1 \mathrm{mmol} / \mathrm{L})$ and from $9.4 \%(79 \mathrm{mmol} / \mathrm{mol})$ to $7.9 \%(63 \mathrm{mmol} / \mathrm{mol})$, respectively, after insulin initiation. ${ }^{6}$ However, the Fremantle study represents patients managed by GPs and specialists. Real world data from the Fenofibrate
Intervention and Event Lowering in Diabetes study of patients with T2DM being managed in primary care settings in Australia, New Zealand, and Finland showed a reduction in median $\mathrm{A}_{1 \mathrm{c}}$ from $8.2 \%(66 \mathrm{mmol} / \mathrm{mol})$ to $7.5 \%(58 \mathrm{mmol} / \mathrm{mol}$; mean difference $-0.5 \%$ ) a year after insulin initiation in patients receiving standard care in a nonintervention arm. ${ }^{7}$ A smaller but more recent study reported reductions in median $\mathrm{A}_{1 \mathrm{c}}$ from $9.9 \%(85 \mathrm{mmol} / \mathrm{mol})$ to $7.3 \%(56 \mathrm{mmol} / \mathrm{mol})$ 24 weeks after initiating insulin in an Australian primary care setting. ${ }^{31}$ These observations suggest that the Diabetes CoStars PSP achieved patient outcomes comparable to those achieved through standard primary care for patients with T2DM in Australia.

The results of the study must be considered in the light of its limitations. First, there was a significant amount of missing data as the collection of $\mathrm{A}_{1 \mathrm{c}}$ values was not mandatory; as such the reported patient outcomes may not be representative of all patients enrolled in the program during the data collection period. Demographic information about patients that may have been the predictors of achievement of FBG targets, such as age, diabetes duration, and other oral antidiabetic drugs, was not available. The details of patient adherence to their titration plan and CDE-led education were also unavailable and it is difficult to interpret the relative contributions of CDE-led support versus the provision of a specific titration plan to patient outcomes in the absence of a comparator group of patients who did not receive CDE-led support. In addition, the brief (12-week) duration of the Diabetes CoStars PSP prevents conclusions being drawn regarding the longterm success in optimizing FBG levels. Further analyses of this dataset should, ideally, include extended follow-up to ascertain whether the initial impact of the program has sustainable benefits over the longer term. Finally, the success of the Diabetes CoStars PSP was measured only in terms of achievement of FBG and $A_{1 c}$ targets. As the program was developed to assist GPs with insulin initiation and titration in recognition of barriers including lack of experience and time to educate patients, it would be interesting to explore whether the Diabetes CoStars PSP has other benefits such as increasing GP confidence in initiating insulin and helping to overcome clinical inertia.

Patient education is an essential component of diabetes care. ${ }^{13,14}$ As specialists in their field, CDEs' knowledge and skills in diabetes education, insulin initiation, and titration are often superior to that of GPs and health professionals recognize that insulin initiation in the primary care setting should be supported by a CDE., ${ }^{9,32}$ The effectiveness of enhanced education and CDE-led support on insulin initiation in Australian 
primary care has recently been reported through the results of a feasibility study into the Stepping Up program. ${ }^{33,34}$ GPs and practice nurses from five centers participated in a practice briefing visit with a CDE and an evening training session with a GP where simple initiation and titration algorithms were provided, as well as up-titration protocols and regular follow-up support. Although only 18 patients were involved in this pilot study, over the course of the program, mean $\mathrm{A}_{1 \mathrm{c}}$ fell from $8.4 \%$ $(68.3 \mathrm{mmol} / \mathrm{mol})$ at baseline to $7.5 \%(58.5 \mathrm{mmol} / \mathrm{mol})$ after 12 weeks of insulin therapy. ${ }^{34}$ While the titration algorithms were reported to be useful and relevant, greater flexibility was identified as a potential improvement to allow the regimens to be adapted to individual patient's circumstances.

The Diabetes CoStars PSP provided flexibility through a choice of titration plan options and improvements in FBG and $A_{1 c}$ were similar regardless of the titration plan selected. In contrast, titration regimen influenced patient outcomes in a multinational randomized trial involving 4,961 patients with T2DM, where reductions in FBG and $\mathrm{A}_{1 \mathrm{c}}$ were greater in patients following an insulin titration protocol in which dose was self-adjusted every 3 days compared with weekly physician-led dose adjustments. ${ }^{19}$ Patients enrolled in the Diabetes CoStars PSP were provided with a titration protocol as well as CDE-led education, suggesting that similar improvements in FBG and $\mathrm{A}_{1 \mathrm{c}}$ can be achieved on any titration plan when support from a CDE is provided.

\section{Conclusion}

Acceptable FBG levels were achieved by $\sim 70 \%$ of patients with T2DM enrolled in the Diabetes CoStars PSP. The Diabetes CoStars PSP was less effective in helping patients achieve target $A_{1 c}$ levels. The results indicate that when insulin initiation in the primary care setting is supported by the provision of titration plans to GPs and CDE-led patient support, patients can achieve similar improvements in FBG and $\mathrm{A}_{1 \mathrm{c}}$ levels regardless of the specific titration plan.

\section{Acknowledgments}

This work has been carried out with financial support from Sanofi Australia Pty Ltd. In compliance with the Uniform Requirements for Manuscripts, established by the International Committee of Medical Journal Editors, the sponsor of this study did not impose any impediment, directly or indirectly, on the publication of the study results. The sponsor reviewed the final manuscript before submission. Statistical analyses were funded by Sanofi Australia Pty Ltd and conducted by Dr Annie Solterbeck of Statistical Revelations Pty Ltd, Melbourne, Australia. Editorial and writing assistance, in accordance with international guidelines for Good Publication Practice (GPP3), was provided by Hazel Palmer (ISMPP CMPPTM) and Adrianna Kalous of Scripitx Pty Ltd, Sydney, Australia; their contributions were funded by Sanofi Australia Pty Ltd.

\section{Author contributions}

All authors were involved in the development of the study protocol. OKL, HS, and MA contributed to data collection. All authors contributed toward data analysis, drafting and critically revising the paper and agree to be accountable for all aspects of the work.

\section{Disclosure}

KMK is an employee of Sanofi Australia Pty Ltd. OKL, HS, and MA are CDEs, they each managed patients involved in the program. They each received an honorarium from the study sponsor for time involved in the development of the study protocol only. AR was an employee of Atlantis Healthcare Pty Ltd, Sydney, Australia; Atlantis Healthcare Pty Ltd was financially reimbursed by Sanofi Australia Pty Ltd for the development and management of the Diabetes CoStars PSP. The authors report no other conflicts of interest in this work.

\section{References}

1. World Health Organization. Global Status Report on Noncommunicable Diseases 2014. Geneva: World Health Organization; 2014.

2. Bralic Lang V, Bergman Markovic B, Kranjcevic K. Family physician clinical inertia in glycemic control among patients with type 2 diabetes. Med Sci Monit. 2015;21:403-411.

3. Mata-Cases M, Benito-Badorrey B, Roura-Olmeda P, et al. Clinical inertia in the treatment of hyperglycemia in type 2 diabetes patients in primary care. Curr Med Res Opin. 2013;29:1495-1502.

4. Khunti K, Damci T, Meneghini L, Pan CY, Yale JF, Group SS. Study of Once Daily Levemir (SOLVE): insights into the timing of insulin initiation in people with poorly controlled type 2 diabetes in routine clinical practice. Diabetes Obes Metab. 2012;14:654-661.

5. Davis J, Chavez B, Juarez DT. Adjustments to diabetes medications in response to increases in hemoglobin a1c: an epidemiologic study. Ann Pharmacother. 2014;48:41-47.

6. Davis TM, Davis WA, Bruce DG. Glycaemic levels triggering intensification of therapy in type 2 diabetes in the community: the Fremantle Diabetes Study. Med J Aust. 2006;184:325-328.

7. Best JD, Drury PL, Davis TM, et al. Glycemic control over 5 years in 4,900 people with type 2 diabetes: real-world diabetes therapy in a clinical trial cohort. Diabetes Care. 2012;35:1165-1170.

8. Furler J, Spitzer O, Young D, Best J. Insulin in general practice-barriers and enablers for timely initiation. Aust Fam Physician. 2011;40: 617-621.

9. Sunaert P, Willems S, Feyen L, et al. Engaging GPs in insulin therapy initiation: a qualitative study evaluating a support program in the Belgian context. BMC Fam Pract. 2014;15:144.

10. Peyrot M, Rubin RR, Khunti K. Addressing barriers to initiation of insulin in patients with type 2 diabetes. Prim Care Diabetes. 2010; 4(Suppl 1):S11-S18. 
11. Cuddihy RM, Philis-Tsimikas A, Nazeri A. Type 2 diabetes care and insulin intensification: is a more multidisciplinary approach needed? Results from the MODIFY survey. Diabetes Educ. 2011;37: 111-123.

12. Brez S, Rowan M, Malcolm J, et al. Transition from specialist to primary diabetes care: a qualitative study of perspectives of primary care physicians. BMC Fam Pract. 2009;10:39.

13. RACGP. General Practice Management of Type 2 Diabetes 2014-2015. Melbourne: RACGP; 2014.

14. American Diabetes Association. Standards of Medical Care in Diabetes 2015. Diabetes Care. 2015;38:S1-S89.

15. Dungan K, Lyons S, Manu K, et al. An individualized inpatient diabetes education and hospital transition program for poorly controlled hospitalized patients with diabetes. Endocr Pract. 2014;20:1265-1273.

16. Walker RJ, Smalls BL, Bonilha HS, Campbell JA, Egede LE. Behavioral interventions to improve glycemic control in African Americans with type 2 diabetes: a systematic review. Ethn Dis. 2013;23:401-408.

17. Tshiananga JK, Kocher S, Weber C, Erny-Albrecht K, Berndt K, Neeser K. The effect of nurse-led diabetes self-management education on glycosylated hemoglobin and cardiovascular risk factors: a meta-analysis. Diabetes Educ. 2012;38:108-123.

18. LaSalle JR, Berria R. Insulin therapy in type 2 diabetes mellitus: a practical approach for primary care physicians and other health care professionals. J Am Osteopath Assoc. 2013;113:152-162.

19. Davies M, Storms F, Shutler S, Bianchi-Biscay M, Gomis R, Group AS. Improvement of glycemic control in subjects with poorly controlled type 2 diabetes: comparison of two treatment algorithms using insulin glargine. Diabetes Care. 2005;28:1282-1288.

20. Diabetes Australia. Diabetes Management in General Practice: Guidelines for Type 2 Diabetes 2012/13 [Internet]. Canberra, ACT: Diabetes Australia; 2012.

21. Phillips P. KISS: 'keep insulin safe and simple' Part 1: initiating insulin in type 2 diabetes. Med Today. 2001;8:23-34.

22. Riddle MC, Rosenstock J, Gerich J, Insulin Glargine Study I. The treatto-target trial: randomized addition of glargine or human NPH insulin to oral therapy of type 2 diabetic patients. Diabetes Care. 2003;26: 3080-3086.

23. Colagiuri S, Dickinson S, Girgis S, Colagiuri R. National Evidence Based Guideline for Blood Glucose Control in Type 2 Diabetes. Canberra: Diabetes Australia and the NHMRC; 2009.

24. Stratton IM, Adler AI, Neil HA, et al. Association of glycaemia with macrovascular and microvascular complications of type 2 diabetes (UKPDS 35): prospective observational study. BMJ. 2000;321: 405-412.
25. Shichiri M, Kishikawa H, Ohkubo Y, Wake N. Long-term results of the Kumamoto Study on optimal diabetes control in type 2 diabetic patients. Diabetes Care. 2000;23(Suppl 2):B21-B29.

26. Ohkubo Y, Kishikawa H, Araki E, et al. Intensive insulin therapy prevents the progression of diabetic microvascular complications in Japanese patients with non-insulin-dependent diabetes mellitus: a randomized prospective 6-year study. Diabetes Res Clin Pract. 1995;28: 103-117.

27. Lin SD, Tsai ST, Tu ST, et al. Glycosylated hemoglobin level and number of oral antidiabetic drugs predict whether or not glycemic target is achieved in insulin-requiring type 2 diabetes. Prim Care Diabetes. 2015;9:135-141.

28. Riddle MC, Vlajnic A, Zhou R, Rosenstock J. Baseline $\mathrm{HbA}_{1 \mathrm{c}}$ predicts attainment of $7.0 \% \mathrm{HbA} 1 \mathrm{c}$ target with structured titration of insulin glargine in type 2 diabetes: a patient-level analysis of 12 studies. Diabetes Obes Metab. 2013;15:819-825.

29. Dailey G. Early and intensive therapy for management of hyperglycemia and cardiovascular risk factors in patients with type 2 diabetes. Clin Ther. 2011;33:665-678.

30. Lovre D, Fonseca V. Benefits of timely basal insulin control in patients with type 2 diabetes. J Diabetes Complications. 2015;29: 295-301.

31. Blackberry ID, Furler JS, Ginnivan LE, et al. An exploratory trial of basal and prandial insulin initiation and titration for type 2 diabetes in primary care with adjunct retrospective continuous glucose monitoring: INITIATION study. Diabetes Res Clin Pract. 2014;106: 247-255.

32. Manski-Nankervis JA, Furler J, Blackberry I, Young D, O'Neal D, Patterson E. Roles and relationships between health professionals involved in insulin initiation for people with type 2 diabetes in the general practice setting: a qualitative study drawing on relational coordination theory. BMC Fam Pract. 2014;15:20.

33. Furler JS, Young D, Best J, et al. Can primary care team-based transition to insulin improve outcomes in adults with type 2 diabetes: the stepping up to insulin cluster randomized controlled trial protocol. Implement Sci. 2014;9:20.

34. Furler JS, Blackberry ID, Walker C, et al. Stepping up: a nurse-led model of care for insulin initiation for people with type 2 diabetes. Fam Pract. 2014;31:349-356.
Pragmatic and Observational Research

\section{Publish your work in this journal}

Pragmatic and Observational Research is an international, peer-reviewed, open access journal that publishes data from studies designed to reflect more closely medical interventions in real-world clinical practice compared with classical randomized controlled trials (RCTs). The manuscript management system is completely online and includes a very quick and fair peer-review

\section{Dovepress}

system. Visit http://www.dovepress.com/testimonials.php to read real quotes from published authors. 